Review

\title{
A Current Overview of Two Viroids That Infect \\ Chrysanthemums: Chrysanthemum stunt viroid and Chrysanthemum chlorotic mottle viroid
}

\author{
Won Kyong Cho, Yeonhwa Jo, Kyoung-Min Jo and Kook-Hyung Kim * \\ Department of Agricultural Biotechnology, Plant Genomics and Breeding Institute, Institute for \\ Agriculture and Life Sciences, College of Agriculture and Life Sciences, Seoul National University, \\ Seoul 151-921, Korea; E-Mails: wonkyong@gmail.com (W.K.C.); yeonhwajo @gmail.com (Y.J.); \\ jokyoungmin@gmail.com (K.-M.J.) \\ * Author to whom correspondence should be addressed; E-Mail: kookkim@snu.ac.kr; \\ Tel.: +82-2-880-4677; Fax: +82-2-873-2317.
}

Received: 1 March 2013; in revised form: 8 April 2013 / Accepted: 8 April 2013 /

Published: 17 April 2013

\begin{abstract}
The chrysanthemum (Dendranthema X grandiflorum) belongs to the family Asteraceae and it is one of the most popular flowers in the world. Viroids are the smallest known plant pathogens. They consist of a circular, single-stranded RNA, which does not encode a protein. Chrysanthemums are a common host for two different viroids, the Chrysanthemum stunt viroid (CSVd) and the Chrysanthemum chlorotic mottle viroid (CChMVd). These viroids are quite different from each other in structure and function. Here, we reviewed research associated with CSVd and CChMVd that covered disease symptoms, identification, host range, nucleotide sequences, phylogenetic relationships, structures, replication mechanisms, symptom determinants, detection methods, viroid elimination, and development of viroid resistant chrysanthemums, among other studies. We propose that the chrysanthemum and these two viroids represent convenient genetic resources for host-viroid interaction studies.
\end{abstract}

Keywords: chrysanthemum; Chrysanthemum stunt viroid (CSVd); Chrysanthemum chlorotic mottle viroid (CChMVd); host-viroid interaction; viroid 


\section{Introduction}

The chrysanthemum (Dendranthema $\mathrm{X}$ grandiflorum) is a member of the family Asteraceae, and it is one of the popular flowers in the world. The international market for cut and potted chrysanthemums is increasing, and chrysanthemums in many European and Asian countries are commercially very important for the floral industry [1]. Several pathogens, including viruses, viroids, and phytoplasma, cause serious diseases in chrysanthemums. To date, nine viruses and two viroids are known to infect chrysanthemums [2].

Viroids are the smallest known plant pathogens. They consist of a circular, single-stranded RNA, which does not encode a protein. Viroid RNAs range from 246 to 401 bases [3,4]. Viroids traffic from cell to cell via plasmodesmata [5]. To date, over 30 species of viroids have been reported; these can be divided into two families, the Pospiviroidae and the Avsunviroidae [6]. The family Pospiviroidae includes five genera, such as Apscaviroid, Cocadviroid, Coleviroid, Hostuviroid, and Pospiviroid. So far, ten species including Chrysanthemum stunt viroid (CSVd) and Potato spindle tuber viroid (PSTVd) are members of the genus Pospiviroid [7]. The genomic RNA of members of the family Pospiviroidae, which replicate in the nucleus, assumes rod-like or quasi-rod-like conformation in which, based on local sequence similarity, five domains have been proposed: the left terminal, pathogenicity, central, variable, and right-terminal domains [8]. Moreover, several conserved regions have been identified in the rod-like conformation, including the central conserved region (CCR), likely involved in replication [9-12], and the terminal conserved region (TCR) or the terminal conserved hairpin (TCH), which appear mutually exclusive [13-15]. The family Avsunviroidae is composed of three genera, such as Avsunviroid, Elaviroid, and Pelamoviroid. Peach latent mosaic viroid (PLMVd) and Chrysanthemum chlorotic mottle viroid (CChMVd) are members of the genus Pelamoviroid. They have highly branched structures with self-cleaving ribozymes, which are not present in the family Pospiviroidae. These viroids replicate in the host chloroplast [16].

Interestingly, chrysanthemums are a common host for two different viroids, the CSVd and the CChMVd, which are quite different from each other in structure and function (Table 1). Here, we summarize and discuss current research associated with CSVd and CChMVd, and we suggest that chrysanthemums and viroids would serve as convenient genetic resources for host-viroid interaction studies.

\section{Symptoms, Isolation, Nucleotide Sequences, and Structures}

Chrysanthemum stunt disease was reported in the early 1950s [17]. It causes light green young leaves, stunting, small leaves and flowers, and reduced rooting ability. Later, infectious material was first isolated from leaves of stunted chrysanthemum, after nucleic acid extraction, centrifugation in sucrose density gradient and electrophoresis in polyacrylamide gels demonstrated the presence of a low molecular weight RNA, which was different from the known PSTVd [17]. This novel viroid, associated with stunt disease in chrysanthemums, was distinct from other known viruses, and it was named CSVd [18]. The complete nucleotide sequence and secondary structure of CSVd was first determined in Australia in 1981 [19]. The identified CSVd comprised 356 bases and displayed about 69\% sequence identity to PSTVd. Subsequently, another CSVd with 354 bases was sequenced [20]. 
Table 1. Characteristics of CSVd and CChMVd viroids.

\begin{tabular}{lll}
\hline Characteristics & CSVd & CChMVd \\
\hline Disease & Chrysanthemum stunt & Chrysanthemum chlorotic mottle \\
\hline Symptoms & $\begin{array}{l}\text { Light green young leaves, chlorotic spots, } \\
\text { stunting, small leaves and flowers, and } \\
\text { decreased rooting ability }\end{array}$ & $\begin{array}{l}\text { Yellow-green mottling, chlorosis, } \\
\text { and dwarfed size }\end{array}$ \\
\hline Family and genus & Pospiviroidae, Pospiviroid & Avsunviroidae, Pelamoviroid \\
\hline Genome size & 354-356 nt & 398-401 nt \\
\hline $\begin{array}{l}\text { Replication } \\
\text { method }\end{array}$ & Asymmetric rolling circle mechanism & $\begin{array}{l}\text { Symmetric rolling circle mechanism } \\
\text { with the hammerhead ribozymes }\end{array}$ \\
\hline $\begin{array}{l}\text { Replication } \\
\text { localization }\end{array}$ & Nucleus & Chloroplast \\
\hline Structure & $\begin{array}{l}\text { Rod-like structure including central } \\
\text { conserved region (CCR) }\end{array}$ & $\begin{array}{l}\text { Branched conformation including } \\
\text { hammerhead ribozymes }\end{array}$ \\
\hline Transmission & Sap, grafting, and seed & Sap, grafting \\
\hline & $\begin{array}{l}\text { Chrysanthemums, Petunia hybrida, tomato, } \\
\text { Gynura aurantiaca, Ageratum, dahlia, }\end{array}$ & \\
& $\begin{array}{l}\text { Senecio, Vinca major, Argyranthemum } \\
\text { frutescens and many plants belonging to the } \\
\text { families Solanaceae and Asteraceae }\end{array}$ & Restricted to chrysanthemums \\
\hline
\end{tabular}

The structures and structural transitions of various viroids, including CSVd, have been determined with thermodynamic, kinetic, and hydrodynamic methods (Figure 1a) [21]. Recently, the sequences of three different CSVd isolates were determined from the US, China, and Australia. Both the US and Australian isolates were composed by several sequence variants, confirming the quasi-species nature of the viroid, but the Chinese isolate consisted of a single variant indicating a low molecular variability for CSVd [22]. Comparative analyses of the nucleotide sequences of $117 \mathrm{CSVd}$ sequence variants revealed nucleotide variations at 103 sites scattered throughout the CSVd genome. However, it is not known whether these nucleotide changes are related to the species specificity of CSVd infections [23]; in addition, possible relationships between nucleotide changes and variant-specific pathogenicity have been recently questioned [23].

Chrysanthemum chlorotic mottle disease was first reported in 1967 in the cultivar "Yellow Delaware" in New York State [24]. This disease could be transmitted by grafting one chrysanthemum to another chrysanthemum cultivar. It causes yellow-green mottling, chlorosis, and dwarf symptoms, but some infected cultivars were asymptomatic [25]. Due to the low abundance of CChMVd in infected plants, compared to the abundance of CSVd, the complete, 398-401 nt sequence of CChMVd was only identified in 1997 (Figure 1b) [26]. Transcripts of CChMVd in vitro were also infectious and caused chlorotic mottle disease [26]. CChMVd can form hammerhead structures in both plus and minus strands, which self-cleave during in vitro and in vivo transcription (Figure 1c) [26]. The hammerhead structures of CChMVd display unique features, including an unpaired A residue after the conserved A9 residue in the plus self-cleaving domain, and an unusually long helix II in the minus one (Figure 1c). The predicted secondary structure of CChMVd is a very stable, branched conformation, which is similar to that of PLMVd. In contrast to viroids with a quasi-rod-like conformation, both 
CChMVd and PLMVd were insoluble in $2 \mathrm{M} \mathrm{LiCl}$ [26]. In one recent study, an in vitro transcript of CChMVd was inoculated to measure the mutation rate of CChMVd, which resulted in being the highest one ever reported in any biological entity [27].

Figure 1. Predicted secondary structures of CSVd and CChMVd. (A) Predicted secondary structure of CSVd was adapted with permission from [22]. The central conserved region (CCR) is indicated by light-red shading and the terminal conserved region is indicated by light-blue shading; (B) Predicted secondary structure of CChMVd was adapted with permission from [28]. Plus and minus self-cleavage domains are delimited by flags, residues conserved in most natural hammerhead structures are boxed, and the self-cleavage sites are indicated by arrows. Light-blue shading and light-red shading in flags, boxes, and arrows refer to plus and minus polarities, respectively. The changes in the tetraloop delimited by positions $82-85$ (UUUC to GAAA) that convert a symptomatic variant into non-symptomatic are shown with green-colored boxes [29]. The light-yellow square demarcates the domain that alternatively can form a kissing-loop interaction [30]; (C) Hammerhead structures of the plus and minus strands of CChMVd were adapted with permission from [28]. Residues conserved in most natural hammerhead structures are on light-blue shading and light-red shading in the plus and minus polarities, respectively, and the self-cleavage sites are indicated by arrows. Blue colored A indicates the position of the extra $\mathrm{A}$ in the CChMVd secondary structure and in its plus hammerhead structure. Numbering is done based on the previous study [28].

A

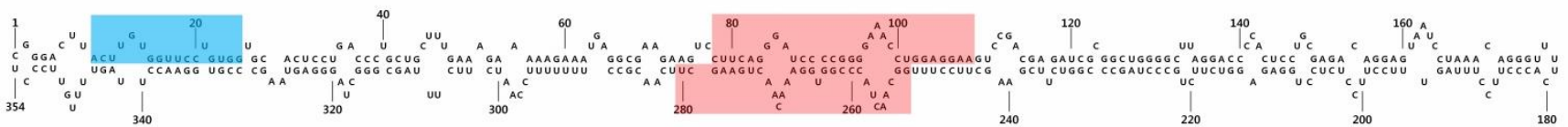

B

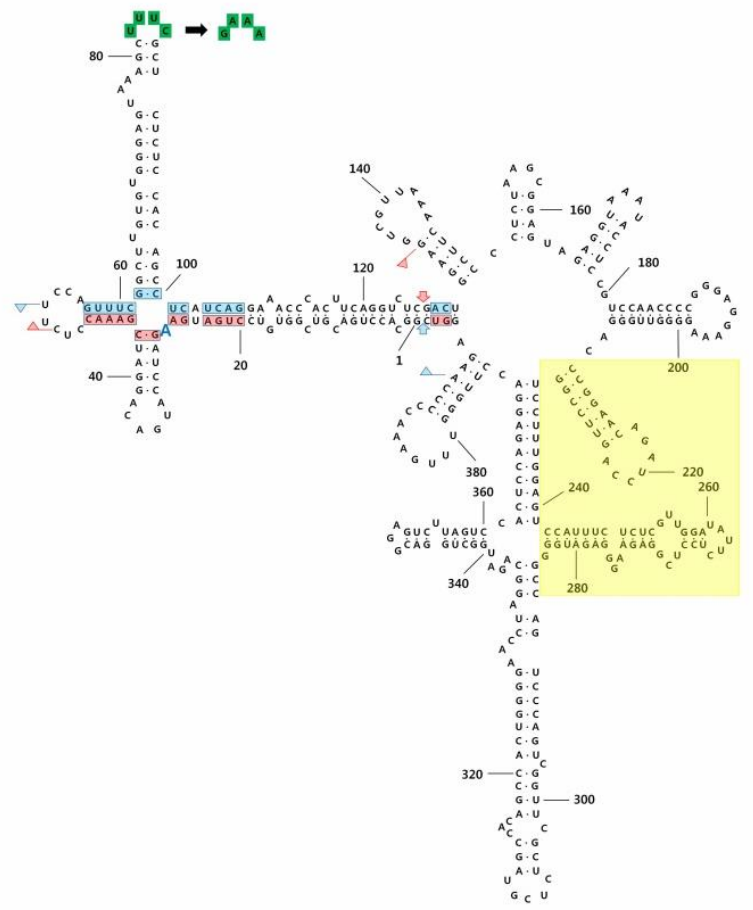

C
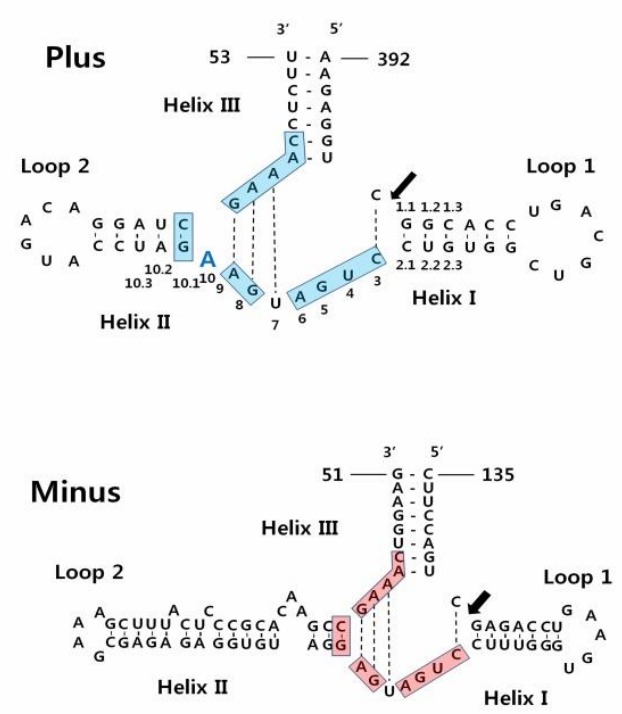


\section{Host Range}

Due to the limited number of studies on viroids, it was thought that CSVd only infected chrysanthemums [31]. Initial reports showed that chrysanthemum plants could serve as host for both CSVd and CChMVd. Moreover, circular and linear forms of CSVd, purified from infected chrysanthemum plants, were infectious when inoculated into Gynura aurantiaca plants [32]. Also, a linear CSVd RNA, synthesized by in vitro transcription, could infect chrysanthemum plants and other plants that belong to the families Solanaceae and Asteraceae, without causing any disease symptoms [33]. Under natural conditions, infections of CSVd have been reported in Petunia hybrida [34], Ageratum, Dahlia [35], and Senecio (cineraria) [36]. CSVd was also identified in Vinca major with reverse transcriptase (RT)-PCR [37]. An infection of CSVd, identified with RT-PCR, was also reported in asymptomatic Argyranthemum frutescens (marguerite daisy) [38,39]. In contrast to the quite wide range identified for $\mathrm{CSVd}$, the known host range of CChMVd is very restricted. For example, of 51 species and cultivars tested, CChMVd was infectious in only two chrysanthemum species [40].

In general, CSVd and CChMVd are mechanically transmitted through tools, like knives and scissors, used on infected chrysanthemums and then on healthy material for grafting and flower cutting. A previous study also demonstrated seed-borne transmission of CSVd in chrysanthemums; in that case, the ambient temperature during a cross could influence the rate of CSVd transmission to progeny [41]. However, CSVd could not be transmitted from infested soil [42], and no insect vector has been reported to transmit it.

\section{Identification of the Two Viroids}

Many large-scale screening studies have been performed to detect CSVd and CChMVd. For instance, a total of 2,480 chrysanthemum samples collected from six Australian states were tested for viroid and virus infections with enzyme-linked, immunosorbent assays (ELISAs) and cDNA probes [43]. As a result, CSVd has been found in mixed infection with other viruses [43].

CSVd is one of several quarantined pathogens in European countries, under order of the European Union's Plant Health Directive (2000/29/EC) [44]. The European Food Safety Authority (EFSA) has released a Scientific Opinion on the assessment of the risk of solanaceous pospiviroids for the EU territory and the identification and evaluation of risk management options [45]. In Italy, to produce healthy chrysanthemum plants, phytosanitary measures, like RT-PCR and hybridization assays, were carried out to test for CSVd infections in 39 seasonal and 93 year-round chrysanthemums [1]. In Slovenia, 12 out of 200 chrysanthemum plants were found to be infected with CSVd [46]. Recently, a study reported the first identification of CSVd in Turkey; where two infected plants out of 154 chrysanthemums tested positive in an RT-PCR assay [47]. Moreover, CSVd was identified by RT-PCR in infected chrysanthemum plants in Egypt [48].

In Asian countries, CSVd and CChMVd have been reported in Japan, Korea, China, and India. Cultivated and wild chrysanthemums were tested for CSVd infections in Japan with RT-PCR [49]. Out of 89 samples, 80 samples were infected with CSVd. In addition, eight wild chrysanthemum species, which did not exhibit any CSVd symptoms, carried CSVd. Among $21 \mathrm{CSVd}$ isolates, a total of five variants were revealed; these variants showed frequent mutations. 
In Akita Prefecture, Japan, CChMVd infections in chrysanthemums caused distinct yellow leaf mottling and necrosis. These symptoms were caused by CChMVd variants containing a UUUC tetraloop (Figure 1b) [50], a structural domain already identified as a specific CChMVd pathogenic determinant [26]. A previous study had detected CChMVd infections in various chrysanthemums grown for three years in Akita Prefecture, revealing that $20 \%$ of chrysanthemums were infected with CChMVd [51]. In natural conditions, chrysanthemums that were only infected with CChMVd variants with the UUUC tetraloop did not show any noticeable disease symptoms [51]. In this case, light and temperature may interfere with symptoms induced by variants containing the symptomatic domain. Furthermore, in Kyoto, CChMVd was also detected in cut chrysanthemums from Japan and the Netherlands; this indicated that CChMVd infections have spread over a wide range in Japan [52].

In Korea, CSVd was first identified in chrysanthemum cv. Chunkwang in 2001. The complete sequences for two isolates (K1 and K2) were determined [53]. Later, a total of 64 commercial chrysanthemum cultivars in Korea were tested for CSVd infections, and the infection rate of each cultivar ranged from $9.7 \%$ to $66.8 \%$ [54]. Furthermore, the complete sequence was reported for the CChMVd-SSHA6 viroid from Korea; this variant caused yellow spots and growth reduction in some infected cultivars [54].

In China, CChMVd was first reported in 2008. In 2010, 13 samples with mild chlorotic spots were collected, and all were infected with CChMVd [55]. In India, chrysanthemum cultivars were screened for CSVd infections with RT-PCR and DNA-RNA hybridization: 70\% of cultivars were infected with CSVd [36].

\section{Replication Mechanisms}

Viroids replicate in vivo via a rolling circle mechanism, which requires a specific cleavage procedure that converts multimeric viroid copies into monomeric forms [56,57]. Viroids, including CSVd, in the family Pospiviroidae replicate in the nucleus via an asymmetric rolling circle mechanism [58]. In contrast, CChMVd and members of the family Avsunviroidae replicate via a symmetric rolling circle mechanism in the chloroplast, mediated by the hammerhead ribozyme. The hammerhead ribozyme in the family Avsunviroidae determines self-cleavage of oligomeric RNAs during viroid replication; replication is further catalyzed by RNA polymerase and RNA ligase [59].

The hammerhead ribozyme of CChMVd has been intensively studied. The plus hammerhead ribozyme of CChMVd contains an additional A (A10) residue between the conserved A9 and the quasi-conserved G10.1 residues (Figure 1c) [28]. The additional A10 residue causes a moderate decrease in the trans-cleaving rate, while substitution of A10 to $\mathrm{C}$ and A10 to $\mathrm{G}$ causes major detrimental effects (Figure 1c). In contrast, the A10 to U substitution increased the trans-cleaving rate by 3-4-fold. Some hammerheads display deviations from the consensus sequence because certain residues might be involved in unknown important functions, other than self-cleavage [28]. In addition, high-pressure experiments identified two different types of CChMVd hammerhead conformation that exhibit fast-cleaving and slow cleaving activities [60].

Interactions between loop 1 and loop 2 of CChMVd hammerheads in both polarity strands (and in most natural hammerheads) have been shown to play major roles in self-clearing activity of these ribozymes [30]. Moreover, site-directed mutagenesis, bioassays, and progeny analysis studies showed 
that a kissing-loop interaction is important for in vitro folding and viroid infectivity (Figure 1b) [61]. For instance, the introduction of a single mutation in the kissing loops led to low or no infectivity, but an introduction of second mutation restoring the kissing-loop interaction resulted in an infectious agent [61]. These results indicated that the loop-loop tertiary interactions are important for viroid infectivity and for the folding and catalytic activity of most natural hammerheads [30].

\section{Symptom Determinants}

Of the two viroids discussed here, CSVd is the more serious pathogen. It leads to reductions in the quality and quantity of chrysanthemum production. However, the sequence regions that confer the different symptoms are currently unknown. In contrast, the functional determinants for pathogenicity have been mapped in CChMVd. A previous study identified a nonpathogenic CChMVd strain that could protect against challenge inoculations with a pathogenic strain. Sequence analysis and site-directed mutagenesis have revealed that a substitution from UUUC to GAAA in the pathogenic CChMVd strain led to a nonpathogenic viroid with normal replication ability (Figure 1b). The identified pathogenicity determinant was located in a tetraloop of the predicted, branched conformation of CChMVd [62]. Later, the tetraloop of CChMVd, was further investigated with site-directed mutagenesis, bioassay, and analyses of the progenies [29]. The authors found that substitution of the tetraloop with a triloop or a pentaloop did not affect the infectivity of CChMVd. However, the thermodynamically stable GAAA tetraloop of the nonpathogenic CChMVd strain could not be replaced with other stable tetraloops of the UNCG family without causing functional changes. These data indicate that the sequence was the major factor that preserved the functional tetraloop motif, rather than the structure. After the introduction of a site-directed modified CChMVd variant into chrysanthemum, this nonpathogenic variant eventually evolved into a pathogenic CChMVd one, which acquired the UUUC tetraloop characteristics. This study provided evidence of the stronger biological fitness of the pathogenic CChMVd with the UUUC tetraloop, compared to that of the nonpathogenic CChMVd with the GAAA tetraloop.

\section{Detection Methods}

Due to the fact that viroids have very low molecular weights, polyacrylamide gel electrophoresis (PAGE) was first used to detect viroid RNAs $[63,64]$. Molecular method to detect and quantify CSVd RNA in infected plants based on hybridization assays, where the viroid RNA hybridized to a ${ }^{32}$ P-labeled cDNA of CSVd, were also developed [65,66]. The ${ }^{32} \mathrm{P}$-labelled cDNA probe that targeted PSTVd was able to detect both PSTVd and CSVd [67]. In addition, several nonradioactive probes were developed, like photobiotin-labeled DNA probes, [68,69]. Both ${ }^{32} \mathrm{P}$-labeled and biotin-labeled cDNA probes were used and compared for the detection of CSVd and PSTVd [69]. The most sensitive method for detecting viroids is probably the RT-PCR-based approach. A previous study developed a simple and rapid method for nucleic acid extraction, without tissue homogenization, for detecting CSVd [70]. Typically, two different methods have been applied for detecting CSVd, either a combination of RT-PCR and hybridization, or a combination of RT-PCR and ELISA [39,70].

To identify several viroids or a novel viroid in a single host, several RT-PCR primer sets or digoxigenin-labeled polyprobes have been developed based on consensus sequences identified in all pospiviroids [71,72]. Also, a one-tube/one-step RT-PCR method was developed to detect seven 
viroids, including CSVd, belonging to four genera [73]. In addition, a multiplex, direct RT-PCR method was developed to detect CSVd and CChMVd in a small amount of plant tissues [74,75]. Furthermore, a previous study developed real-time PCR (TaqMan ${ }^{\circledR}$ ) assays for detecting six viroids, including CSVd, infecting solanaceous hosts [44]. Recently, a multiplex RT-PCR primer set was developed to detect viruses and viroids, including Chrysanthemum virus B, Tomato aspermy virus, CSVd, and CChMVd in chrysanthemums [76]. These assays might be useful for detecting different viroids infecting the same host simultaneously. These methods could be applied to quarantine, certification, and screening purposes.

\section{Efforts to Eliminate Viroids from Infected Plants}

Several attempts have been made to generate viroid-free plants. First, a long-term heat treatment at $35{ }^{\circ} \mathrm{C}$ for $14-37$ weeks was proposed for treating CSVd-infected plants [77]. In contrast, a cold treatment was shown to be effective for eliminating CSVd in infected chrysanthemum plants [78]. Those authors grew CSVd-infected chrysanthemum plants at $5{ }^{\circ} \mathrm{C}$ for six months; subsequently, the meristem tips of cold-treated chrysanthemums were used to generate non-infected chrysanthemum plants [78]. Another study found that promoting vegetative propagation during a low-temperature period could eliminate CSVd from the infected chrysanthemums [42]. However, it was also shown that, after reducing the level of CSVd RNA in the plant during a low-temperature period, CSVd replication could again increase when the infected plants were grown at normal or high temperatures [79].

Recently, a Japanese research group established a new approach for obtaining CSVd-free chrysanthemums with leaf primordium-free, shoot apical meristems (LP-free SAMs). The attachment of LP-free SAMs to the root tips of CSVd-free chrysanthemums or cabbage resulted in the production of $14 \%$ and $3 \%$ CSVd-free plants, respectively [80]. A previous study generated CSVd-free and CSVd-infected plants by culturing different-sized SAMs dissected from the "Piato" plant; the authors reported that CSVd-infected plants flowered, even in the long-day condition [81]. These data suggested that CSVd might induce autonomous flowering in chrysanthemum, which is known to be a qualitative, short-day flowering plant [81]. In the same way, this research group has regenerated CChMVd-free chrysanthemums [52]. To date, the elimination of viroids from infected plants has remained a challenge.

\section{Identification and Development of Viroid-Resistant Chrysanthemum Cultivars}

Most cultivated chrysanthemums are known to be infected with CSVd, which even if asymptomatic, could transfer CSVd to other susceptible cultivars in fields. Controlling CSVd with chemical treatments and culturing approaches is currently a challenging endeavor. Therefore, the ideal would be to find chrysanthemum cultivars resistant to CSVd, for developing new CSVd-resistant chrysanthemum lines. In a recent study, 35 chrysanthemum lines, including commercial cultivars, wild species, and interspecific hybrids, were screened to identify resistant plants by exposing uninfected plants to CSVd-infected plants with a grafting system [82]. RT-PCR was routinely performed to check for CSVd infections in the upper leaves of the scion every month after grafting. Of the 35 lines, the "Okayamaheiwa" cultivar showed strong resistance against CSVd. After crossing the "Okayamaheiwa" cultivar with two other susceptible cultivars, "Sei-elza" and "Anri," 76 and 8 F1 progeny individuals 
were produced. Among the F1 progeny, 14 were not infected with CSVd, thus indicating that CSVd resistance was achieved in the first hybrid generation [82]. CSVd was not present in the SAMs or LPs of resistant chrysanthemum cultivars after grafting. Taking the absence of CSVd in the SAMs and LPs as a marker, 20 cultivars were identified as CSVd resistant out of 85 commercial cultivars tested; however, later grafting experiments showed that CSVd was detected in two of these candidate resistant cultivars [83].

Another previous study examined six cultivars, and identified a cultivar named "Utage," which exhibited a reduced level of CSVd replication. A total of 67 cultivars, obtained from the self-pollination of "Utage," were tested for CSVd resistance by RT-PCR. Of those, three plants (C7, A30, and A27) were found to have strong CSVd resistance [84].

Transgenic chrysanthemum lines were generated where a specific double-stranded RNA ribonuclease gene (pacl) of Schizosaccharomyces pombe was introduced with Agrobacterium-mediated transformation $[85,86]$. The generated transgenic plant, which expressed pac1, exhibited reduced levels of viroid infection and accumulation [85]. In addition, these plants were resistant to Tomato spotted wilt virus (TSWV). When hybrid lines were crossed with the transgenic line or with a wild chrysanthemum species, the progeny displayed either resistance or high susceptibility to TSWV infections. This study demonstrated the utility of $\mathrm{pacl}$ for achieving both viroid and virus resistance.

\section{Other Studies Associated with CSVd and CChMVd}

Cross-protection can be defined as the interference in symptom expression generated by a previously inoculated viroid against subsequent infection by other strains of the same or a closely related viroid [87]. Five different viroids have been used for cross-protection studies in tomato and chrysanthemum plants [87]. Each viroid displayed different symptoms in chrysanthemum plants and, interestingly, three of them, CSVd, a mild PSTVd strain, and a severe PSTVd strain, could protect against Citrus exocortis viroid (CEVd); in contrast, CChMVd could not protect against the severe PSTV strain, CEVd, or CSVd, even though the challenge-inoculated viroid had replicated. Thus, viroid-infected plants showing attenuated symptoms might induce cross-protection against other viroids. Moreover it has been shown that non-symptomatic strain of CChMVd induces cross-protection against the symptomatic strain of the same viroid species [62].

Virus and viroid infections generate small interfering RNAs that interact with the RNA-induced silencing complex (RISC) of the host. A previous study demonstrated the presence of small RNAs (22 nt) derived from CSVd by northern blot analysis accumulating in similar amounts in different plants, regardless of differences in symptoms [88]. In addition, small interfering RNAs may be involved in the inhibition of enzyme activity; for example, the stunted phenotype of CSVd-infected plants might be associated with decreased levels of the plant growth hormone gibberelic acid (GA), due to disturbed GA 20-oxidase activity [89].

\section{Future Directions in Host-Viroid Interaction Studies}

Compared to studies on plant viruses, studies on viroids are very limited and mostly restricted to their detection and identification. In particular, host factors involved in viroid replication, pathogenicity, and movement have not been well characterized. For studying host-viroid interactions, 
chrysanthemum interactions with CSVd and CChMVd have several advantages. First, the two viroids are quite different from each other in replication mechanisms and structure. They belong to two different viroid families, but they do have a common host: chrysanthemums. Second, several proteinRNA interaction techniques will lead to the identification of chrysanthemum proteins that might function for both viroids, while others might be specific for either CSVd or CChMVd. In addition, next-generation sequencing-based approaches might provide information on the chrysanthemum transcriptome and how it is regulated by the two different viroids. Chrysanthemum plants are easily transformed, and creating transgenic plants is not difficult. However, there are several obstacles to using the chrysanthemum as a host, including the finding of a viroid-free, diploid chrysanthemum, because most chrysanthemums are infected with viroids and many are polyploid.

Currently, our research group is working to establish a model system for host-viroid interactions with transcriptomic approaches. We anticipate that host transcript RNA profiling studies will reveal several host candidate genes involved in chrysanthemum-viroid interactions. Characterization of host gene functions will provide important information in understanding viroid disease processes, which may facilitate the development of new methods for their control.

\section{Acknowledgments}

This research was supported, in part, by grants from the National Research Foundation of Korea (NRF), a grant funded by the Korean government (MEST) (No. 2011-0010027); the Next-Generation BioGreen 21 Program (No. PJ00798402), through the Rural Development Administration; and the Vegetable Breeding Research Center (No. 710001-03), through the Agriculture Research Center program from the Ministry for Food, Agriculture, Forestry, and Fisheries. The authors would like to thank the two anonymous reviewers for their helpful comments.

\section{Conflict of Interest}

The authors declare no conflict of interest.

\section{References and Notes}

1. Tomassoli, L.; Faggioli, F.; Zaccaria, A.; Caccia, R.; Albani, M.; Barba, M. Molecular diagnosis of Chrysanthemum stunt viroid for routine indexing. Phytopathol. Mediterr. 2004, 43, 285-288.

2. Verma, N.; Sharma, A.; Ram, R.; Hallan, V.; Zaidi, A.A.; Garg, I.D. Detection, identification and incidence of Chrysanthemum B carlavirus in chrysanthemum in India. Crop Prot. 2003, 22, 425-429.

3. Ding, B. Viroids: Self-replicating, mobile, and fast-evolving noncoding regulatory RNAs. Wiley Interdiscip. Rev. RNA 2010, 1, 362-375.

4. Navarro, B.; Gisel, A.; Rodio, M.E.; Delgado, S.; Flores, R.; di Serio, F. Viroids: How to infect a host and cause disease without encoding proteins. Biochimie 2012, 94, 1474-1480.

5. Ding, B.; Wang, Y. Viroids: Uniquely simple and tractable models to elucidate regulation of cell-to-cell trafficking of RNA. DNA Cell. Biol. 2009, 28, 51-56.

6. Góra-Sochacka, A. Viroids: Unusual small pathogenic RNAs. Acta Biochim. Pol. 2004, 51, 587-608. 
7. Owens, R.A.; Flores, R.; Di Serio, F.; Li, S.F.; Pallás, V.; Randles, J.W.; Sano, T.; Vidalakis, G. Viroids. In Virus Taxonomy: Ninth Report of the International Committee on Taxonomy of Viruses; Andrew, M.Q.K., Michael, J.A., Eric, B.C., Elliot, J.L., Eds.; Elsevier Academic Press: London, UK, 2012; pp. 1221-1234.

8. Keese, P.; Symons, R.H. Domains in viroids: Evidence of intermolecular RNA rearrangements and their contribution to viroid evolution. Proc. Natl. Acad. Sci. USA 1985, 82, 4582-4586.

9. McInnes, J.; Symons, R.H. Comparative Structure of Viroids and Their Rapid Detection using Radioactive and Nonradioactive Nucleic Acid Probes. In Viroids and Satellites: Molecular Parasites at the Frontier of Life; Maramorosch, K., Ed.; CRC Press: Boca Raton, FL, USA, 1991; pp. 21-58.

10. Baumstark, T.; Schröder, A.R.; Riesner, D. Viroid processing: Switch from cleavage to ligation is driven by a change from a tetraloop to a loop E conformation. EMBO J. 1997, 16, 599-610.

11. Gas, M.-E.; Hernández, C.; Flores, R.; Daròs, J.-A. Processing of nuclear viroids in vivo: An interplay between RNA conformations. PLoS Pathog. 2007, 3, e182.

12. Gas, M.-E.; Molina-Serrano, D.; Hernández, C.; Flores, R.; Daròs, J.-A. Monomeric linear RNA of citrus exocortis viroid resulting from processing in vivo has 5'-phosphomonoester and 3'-hydroxyl termini: Implications for the RNase and RNA ligase involved in replication. J. Virol. 2008, 82, 10321-10325.

13. Koltunow, A.M.; Rezaian, M.A. Grapevine yellow speckle viroid: Structural features of a new viroid group. Nucleic Acids Res. 1988, 16, 849-864.

14. Puchta, H.; Ramm, K.; Sänger, H.L. The molecular structure of hop latent viroid (HLV), a new viroid occurring worldwide in hops. Nucleic Acids Res. 1988, 16, 4197-4216.

15. Flores, R.; Di Serio, F.; Hernández, C. Viroids: The Noncoding Genomes, Seminars in Virology, 1997; Elsevier: Waltham, MA, USA,1997; pp. 65-73.

16. Ding, B. The biology of viroid-host interactions. Annu. Rev. Phytopathol. 2009, 47, 105-131.

17. Diener, T.; Lawson, R. Chrysanthemum stunt: A viroid disease. Virology 1973, 51, 94-101.

18. Diener, T.O. Viroids: The smallest known agents of infectious disease. Annu. Rev. Microbiol. 1974, 28, 23-40.

19. Haseloff, J.; Symons, R.H. Chrysanthemum stunt viroid: Primary sequence and secondary structure. Nucleic Acids Res. 1981, 9, 2741-2752.

20. Gross, H.J.; Krupp, G.; Domdey, H.; Raba, M.; Jank, P.; Lossow, C.; Alberty, H.; Ramm, K.; Sanger, H.L. Nucleotide sequence and secondary structure of citrus exocortis and chrysanthemum stunt viroid. Eur. J. Biochem. 1982, 121, 249-257.

21. Sieger, G.; Hofmann, H.; Förtsch, J.; Gross, H.J.; Randies, J.W.; Sänger, H.L.; Riesner, D. Conformational transitions in viroids and virusoids: Comparison of results from energy minimization algorithm and from experimental data. J. Biomol. Struct. Dyn. 1984, 2, 543-571.

22. Yoon, J.Y.; Palukaitis, P. Sequence comparisons of global Chrysanthemum stunt viroid variants: Multiple polymorphic positions scattered through the viroid genome. Virus Genes 2012, 46, 97-104.

23. Yoon, J.; Baek, E.; Palukaitis, P. Are there strains of Chrysanthemum stunt viroid? J. Plant Pathol. 2012, 94, 697-701.

24. Dimock, A.W.; Geissinger, C.M. A newly recognized disease of chrysanthemum caused by a graft-transmissible agent. Phytopathology 1969, 59, 1024 (abstr.). 
25. Dimock, A.W.; Geissinger, C.M.; Horst, R.K. Chlorotic mottle: A newly recognized disease oí chrysanthemum. Phytopathology 1971, 61, 415-419.

26. Navarro, B.; Flores, R. Chrysanthemum chlorotic mottle viroid: Unusual structural properties of a subgroup of self-cleaving viroids with hammerhead ribozymes. Proc. Natl. Acad. Sci. USA 1997, 94, 11262-11267.

27. Gago, S.; Elena, S.F.; Flores, R.; Sanjuán, R. Extremely high mutation rate of a hammerhead viroid. Science 2009, 323, 1308-1308.

28. De la Peña, M.; Flores, R. An extra nucleotide in the consensus catalytic core of a viroid hammerhead ribozyme: Implication for the design of more efficient ribozymes. J. Biol. Chem. 2001, 276, 34586-34593.

29. De la Pena, M.; Flores, R. Chrysanthemum chlorotic mottle viroid RNA: Dissection of the pathogenicity determinant and comparative fitness of symptomatic and non-symptomatic variants. J. Mol. Biol. 2002, 321, 411-421.

30. Dufour, D.; De La Peña, M.; Gago, S.; Flores, R.; Gallego, J. Structure-function analysis of the ribozymes of Chrysanthemum chlorotic mottle viroid: A loop-loop interaction motif conserved in most natural hammerheads. Nucleic Acids Res. 2009, 37, 368-381.

31. Runia, W.T.; Peters, D. The response of plant species used in agriculture and horticulture to viroid infections. Eur. J. Plant. Pathol. 1980, 86, 135-146.

32. Palukaitis, P.; Symons, R.H. Purification and characterization of the circular and linear forms of chrysanthemum stunt viroid. J. Gen. Virol. 1980, 46, 477-489.

33. Matsushita, Y.; Penmetcha, K.K.R. In vitro-transcribed Chrysanthemum stunt viroid RNA is infectious to chrysanthemum and other plants. Phytopathology 2009, 99, 58-66.

34. Verhoeven, J.T.J.; Arts, M.S.J.; Owens, R.A.; Roenhorst, J.W. Natural infection of petunia by chrysanthemum stunt viroid. Eur. J. Plant. Pathol. 1998, 104, 383-386.

35. Nakashima, A.; Hosokawa, M.; Maeda, S.; Yazawa, S. Natural infection of Chrysanthemum stunt viroid in dahlia plants. J. Gen. Plant. Pathol. 2007, 73, 225-227.

36. Singh, D.; Pathania, M.; Ram, R.; Zaidi, A.A.; Verma, N. Screening of chrysanthemum cultivars for Chrysanthemum stunt viroid in an Indian scenario. Arch. Phytopathol. Plant. Protect. 2010, $43,1517-1523$.

37. Nie, X.; Singh, R.P.; Bostan, H. Molecular cloning, secondary structure, and phylogeny of three pospiviroids from ornamental plants. Can. J. Plant Pathol. 2005, 27, 592-602.

38. Torchetti, E.M.; Navarro, B.; Trisciuzzi, V.N.; Nuccitelli, L.; Silletti, M.R.; Di Serio, F. First report of Chrysanthemum stunt viroid in Argyranthemum frutescens in Italy. J. Plant. Pathol. 2012, 94, 451-454.

39. Menzel, W.; Maiss, E. Detection of Chrysanthemum stunt viroid (CSVd) in cultivars of Argyranthemum frutescens by RT-PCR-ELISA. Z. Pflanzenkr. Pflanzenschutz 2000, 107, 548-552.

40. Horst, R.K. Chrysanthemum chlorotic mottle. In The Viroids, The Viruses series; Plenum Press: New York, NY, USA, 1987; Volume 11, pp. 291-295.

41. Chung, B.N.; Pak, H.S. Seed transmission of Chrysanthemum stunt viroid in chrysanthemum. Plant Pathol. J. 2008, 24, 31-35.

42. Sugiura, H.; Hanada, K. Chrysanthemum stunt viroid, a disease of large-flowered chrysanthemum [Dendranthema] in Niigata prefecture [Japan]. J. Japan. Soc. Hort. Sci. 1998, 67, 432-438. 
43. Hill, M.F.; Giles, R.J.; Moran, J.R.; Hepworth, G. The incidence of chrysanthemum stunt viroid, chrysanthemum B carlavirus, tomato aspermy cucumovirus and tomato spotted wilt tospovirus in Australian chrysanthemum crops. Australas. Plant Pathol. 1996, 25, 174-178.

44. Monger, W.; Tomlinson, J.; Booonham, N.; Marn, M.V.; Plesko, I.M.; Molinero-Demilly, V.; Tassus, X.; Meekes, E.; Toonen, M.; Papayiannis, L. Development and inter-laboratory evaluation of real-time PCR assays for the detection of pospiviroids. J. Virol. Methods 2010, 169, 207-210.

45. Baker, R.; Candresse, T.; Dormannsné Simon, E.; Gilioli, G.; Grégoire, J.-C.; Jeger, M.J.; Karajova, O.E.; Lövei, G.; Makowski, D.; Manceau, C.; et al. Scientific opinion on the assessment of the risk of solanaceous pospiviroids for the EU territory and the identification and evaluation of risk management options: Scientific Opinion; EFSA Panel on Plant Health (PLH). EFSA J. 2011, 9, 2330.

46. Mehle, N.; Seljak, G.; Verhoeven, J.T.J.; Jansen, C.C.C.; Prezelj, N.; Ravnikar, M. Chrysanthemum stunt viroid newly reported in Slovenia. Plant Pathol. 2010, 59, 1159-1159.

47. Bostan, H.; Gazel, M.; Elibuyuk, I.O. Occurrence of Pospiviroid in potato, tomato and some ornamental plants in Turkey. Afr. J. Biotechnol. 2012, 9, 2613-2617.

48. El-Dougdoug, K.A.; Rezk, A.A.; Rehab, D.A.; Sofy, A.R. Partially nucleotide sequence and secondary structure of Chrysanthemum stunt viroid Egyptian isolate from infected-chrysanthemum plants. Int. J. Virol. 2012, 8, 133-150.

49. Matsushitali, Y. Nucleotide sequences and distribution of Chrysanthemum stunt viroid in Japan. J. Japan. Soc. Hort. Sci. 2007, 76, 333-337.

50. Yamamoto, H.; Sano, T. Occurrence of Chrysanthemum chlorotic mottle viroid in Japan. J. Gen. Plant. Pathol. 2005, 71, 156-157.

51. Yamamoto, H.; Sano, T. An epidemiological survey of Chrysanthemum chlorotic mottle viroid in Akita Prefecture as a model region in Japan. J. Gen. Plant. Pathol. 2006, 72, 387-390.

52. Hosokawa, M.; Matsushita, Y.; Ohishi, K.; Yazawa, S. Elimination of Chrysanthemum chlorotic mottle viroid (CChMVd) recently detected in Japan by leaf-primordia free shoot apical meristem culture from infected cultivars. J. Japan. Soc. Hort. Sci. 2005, 74, 386-391.

53. Chung, B.N.; Choi, G.S.; Kim, H.R.; Kim, J.S. Chrysanthemum stunt viroid in Dendranthema grandiflorum. Plant Pathol. J. 2001, 17, 194-200.

54. Chung, B.N.; Lim, J.H.; Choi, S.Y.; Kim, J.S.; Lee, E.J. Occurrence of Chrysanthemum stunt viroid in chrysanthemum in Korea. Plant Pathol. J. 2005, 21, 377-382.

55. Zhang, Z.X.; Pan, S.; Li, S.F. First Report of Chrysanthemum chlorotic mottle viroid in Chrysanthemum in China. Plant. Dis. 2011, 95, 1320.

56. Flores, R.; Daròs, J.-A.; Hernández, C. Avsunviroidae family: Viroids containing hammerhead ribozymes. Adv. Virus Res. 2000, 55, 271-323.

57. Flores, R.; Hernández, C.; Alba, A.E.M.d.; Daròs, J.-A.; Serio, F.D. Viroids and viroid-host interactions. Annu. Rev. Phytopathol. 2005, 43, 117-139.

58. Branch, A.D.; Robertson, H.D. A replication cycle for viroids and other small infectious RNA's. Science 1984, 223, 450-455.

59. Flores, R.; Navarro, J.A.; Pena, M.; Navarro, B.; Ambrós, S.; Vera, A. Viroids with hammerhead ribozymes: Some unique structural and functional aspects with respect to other members of the group. Biol. Chem. 1999, 380, 849-854. 
60. Kaddour, H.; Vergne, J.; Hervé, G.; Maurel, M.C. High-pressure analysis of a hammerhead ribozyme from Chrysanthemum chlorotic mottle viroid reveals two different populations of selfcleaving molecule. FEBS J. 2011, 278, 3739-3747.

61. Gago, S.; De la Peña, M.; Flores, R. A kissing-loop interaction in a hammerhead viroid RNA critical for its in vitro folding and in vivo viability. RNA 2005, 11, 1073-1083.

62. De la Peña, M.; Navarro, B.; Flores, R. Mapping the molecular determinant of pathogenicity in a hammerhead viroid: A tetraloop within the in vivo branched RNA conformation. Proc. Natl. Acad. Sci. USA 1999, 96, 9960-9965.

63. Mosch, W.H.M.; Huttinga, H.; Hakkaart, F.A.; De Bokx, J.A. Detection of chrysanthemum stunt and potato spindle tuber viroids by polyacrylamide gelelectrophoresis. Eur. J. Plant Pathol. 1978, $84,85-93$.

64. Morris, T.; Smith, E. Potato spindle tuber disease: Procedures for the detection of viroid RNA and certification of disease-free potato tubers. Phytopathology 1977, 67, 145-150.

65. Palukaitis, P.; Symons, R.H. Hybridization analysis of Chrysanthemum stunt viroid with complementary DNA and the quantitation of viroid RNA sequences in extracts of infected plants. Virology 1979, 98, 238-245.

66. Candresse, T.; Macquaire, G.; Monsion, M.; Dunez, J. Detection of Chrysanthemum stunt viroid (CSVd) using nick translated probes in a dot-blot hybridization assay. J. Virol. Methods 1988, 20, 185-193.

67. Macquaire, G.; Monsion, M.; Mouches, C.; Candresse, T.; Dunez, J. Spot hybridization: Application to viroid identification. Ann. Inst. Pasteur. Virol. 1984, 135, 219-230.

68. McInnes, J.L.; Habili, N.; Symons, R.H. Nonradioactive, photobiotin-labelled DNA probes for routine diagnosis of viroids in plant extracts. J. Virol. Methods 1989, 23, 299-312.

69. Candresse, T.; Macquaire, G.; Brault, V.; Monsion, M.; Dunez, J. ${ }^{32} \mathrm{P}$-and biotin-labelled in vitro transcribed cRNA probes for the detection of Potato spindle tuber viroid and Chrysanthemum stunt viroid. Res. Virol. 1990, 141, 97-107.

70. Nakahara, K.; Hataya, T.; Uyeda, I. A simple, rapid method of nucleic acid extraction without tissue homogenization for detecting viroids by hybridization and RT-PCR. J. Virol. Methods 1999, 77, 47-58.

71. Bostan, H.; Nie, X.; Singh, R.P. An RT-PCR primer pair for the detection of Pospiviroid and its application in surveying ornamental plants for viroids. J. Virol. Methods 2004, 116, 189-193.

72. Torchetti, E.M.; Navarro, B.; Di Serio, F. A single polyprobe for detecting simultaneously eight pospiviroids infecting ornamentals and vegetables. J. Virol. Methods 2012, 186, 141-146.

73. Ragozzino, E.; Faggioli, F.; Barba, M. Development of a one tube-one step RT-PCR protocol for the detection of seven viroids in four genera: Apscaviroid, Hostuviroid, Pelamoviroid and Pospiviroid. J. Virol. Methods 2004, 121, 25-29.

74. Hosokawa, M.; Shiba, H.; Kawabe, T.; Nakashima, A.; Yazawa, S. A simple and simultaneous detection method for two different viroids infecting chrysanthemum by multiplex direct RT-PCR. J. Japan. Soc. Hort. Sci. 2007, 76, 60-65.

75. Hosokawa, M.; Matsushita, Y.; Uchida, H.; Yazawa, S. Direct RT-PCR method for detecting two chrysanthemum viroids using minimal amounts of plant tissue. J. Virol. Methods 2006, 131, 28-33. 
76. Song, A.; You, Y.; Chen, F.; Li, P.; Jiang, J.; Chen, S. A multiplex RT-PCR for rapid and simultaneous detection of viruses and viroids in chrysanthemum. Lett. Appl. Microbiol. 2013, 56, $8-13$.

77. Hollings, M.; Stone, O.M. Attempts to eliminate chrysanthemum stunt from chrysanthemum by meristem-tip culture after heat-treatment. Ann. Appl. Biol. 1970, 65, 311-315.

78. Paduch-Cichal, E.; Kryczyński, S. A low temperature therapy and meristem-tip culture for eliminating four viroids from infected plants. J. Phytopathol. 1987, 118, 341-346.

79. Chung, B.N.; Huh, E.J.; Kim, J.S. Effect of temperature on the concentration of Chrysanthemum stunt viroid in CSVd-infected chrysanthemum. Plant Pathol. J. 2006, 22, 152-154.

80. Hosokawa, M.; Otake, A.; Ohishi, K.; Ueda, E.; Hayashi, T.; Yazawa, S. Elimination of Chrysanthemum stunt viroid from an infected chrysanthemum cultivar by shoot regeneration from a leaf primordium-free shoot apical meristem dome attached to a root tip. Plant Cell Rep. 2004, 22, 859-863.

81. Hosokawa, M.; Ueda, E.; Ohishi, K.; Otake, A.; Yazawa, S. Chrysanthemum stunt viroid disturbs the photoperiodic response for flowering of chrysanthemum plants. Planta 2004, 220, 64-70.

82. Matsushita, Y.; Aoki, K.; Sumitomo, K. Selection and inheritance of resistance to Chrysanthemum stunt viroid. Crop Prot. 2012, 35, 1-4.

83. Nabeshima, T.; Hosokawa, M.; Yano, S.; Ohishi, K.; Doi, M. Screening of chrysanthemum cultivars with resistance to Chrysanthemum stunt viroid. J. Japan. Soc. Hort. Sci. 2012, 81, 285-294.

84. Omori, H.; Hosokawa, M.; Shiba, H.; Shitsukawa, N.; Murai, K.; Yazawa, S. Screening of chrysanthemum plants with strong resistance to chrysanthemum stunt viroid. J. Japan. Soc. Hort. Sci. 2009, 78, 350-355.

85. Ogawa, T.; Toguri, T.; Kudoh, H.; Okamura, M.; Momma, T.; Yoshioka, M.; Kato, K.; Hagiwara, Y.; Sano, T. Double-stranded RNA-specific ribonuclease confers tolerance against Chrysanthemum stunt viroid and Tomato spotted wilt virus in transgenic chrysanthemum plants. Breeding Sci. 2005, 55, 49-55.

86. Toguri, T.; Ogawa, T.; Kakitani, M.; Tukahara, M.; Yoshioka, M. Agrobacterium-mediated transformation of chrysanthemum (Dendranthema grandiflora) plants with a disease resistance gene (pac1). Plant Biotech. 2003, 20, 121-127.

87. Niblett, C.L.; Dickson, E.; Fernow, K.H.; Horst, R.K.; Zaitlin, M. Cross protection among four viroids. Virology 1978, 91, 198-203.

88. Chung, B.N.; Choi, G.S.; Kim, K.T. Chrysanthemum stunt viroid induces the accumulation of small RNAs associated with RNA silencing in infected chrysanthemum. Plant Pathol. J. 2006, 22, 235-238.

89. Huh, E.J.; Lee, S.K.; Chung, B.N.; Lee, I.J.; Choi, S.Y. Changes of growth and gibberellin contents in chrysanthemum by infection of Chrysanthemum stunt viroid. Hort. Environ. Biotech. 2006, 47, 366-370.

(C) 2013 by the authors; licensee MDPI, Basel, Switzerland. This article is an open access article distributed under the terms and conditions of the Creative Commons Attribution license (http://creativecommons.org/licenses/by/3.0/). 